\title{
Publisher's Note: Cascading failures in interdependent networks with finite functional components [Phys. Rev. E 94, 042304 (2016)]
}

M. A. Di Muro, S. V. Buldyrev, H. E. Stanley, and L. A. Braunstein

(Received 29 November 2016; published 5 December 2016)

DOI: 10.1103/PhysRevE.94.069901

This paper was published online on 5 October 2016 with errors in the text on page 3 , and in the Acknowledgments. On page 3, right-hand column, the third line after Eq. (26) should read as " $8 / 3<\lambda<3$ "; the fourth and fifth lines should read as " $2+2 /(m-1)<\lambda<2+2 /(m-2)$." In the Acknowledgments, the first sentence should read as "The Boston University and the Yeshiva University work was supported by DTRA Grant No. HDTRA1-14-1-0017; ...." The paper has been corrected as of 29 November 2016. The text is incorrect in the printed version of the journal. 